\title{
The role of re-acceleration in the understanding of Cosmic-Ray direct and indirect data.
}

\section{Martina Cardillo*}

Istituto di Astrofisica e Planetologia Spaziali-INAF

E-mail: martina.cardillo@inaf.it

In the last years, the improvement of performances of high-energy instruments provided a great amount of data relating to Cosmic-Rays. In particular, in the $\gamma$-ray band, we had the first evidence of CR energization at Supernova Remnant shocks. Several models were developed in order to isolate the hadronic component from the leptonic one and to find the first direct proof of CR acceleration in those sources. Because of some spectral features in disagreement with the Diffusive Shock Acceleration theory, however, the role of pre-existing CR re-acceleration was taken into account, finding that not only its contribution is not negligible but also that it could explain some important characteristics of CR particle and $\gamma$-ray spectra.

36th International Cosmic Ray Conference -ICRC2019-

July 24th - August 1st, 2019

Madison, WI, U.S.A.

${ }^{*}$ Speaker. 


\section{Introduction}

Cosmic Ray (CR) particles are mainly protons and heavy nuclei with an energy spectrum extended from some $\mathrm{GeV}$ to $10^{20} \mathrm{eV}$. They follow a power-law distribution with several important features: a bending at the lowest energies due to solar modulation, a steepening of the spectrum from $E^{-2.7}$ to $E^{-3.1}$ at $E \sim 10^{15} \mathrm{eV}$ (knee) and a hardening at energies of about $10^{18} \mathrm{eV}$ (ankle) $[15,8,27$, for a review]. We know that these particles are accelerated through the first order Fermi mechanism (or diffusive shock acceleration, DSA) $[24,25,10]$ but the issue of their origin is still open to discussion. Focusing our attention on Galactic CRs $\left(E<10^{15} \mathrm{eV}\right)$, Supernova Remnants (SNRs) are considered their main sources because only $10 \%$ of the SN explosion energy is sufficient to explain the CR Galactic energy density $\left(W_{C R} \sim 1 \mathrm{eV} / \mathrm{cm}^{3}\right)$.

These objects are perfect locations for DSA, but we are still looking for direct evidences of $\mathrm{CR}$ acceleration in their shocks that can be obtained only in the $\gamma$-ray energy band. Neutral pions produced in the proton-proton (pp) interactions, indeed, decay in two $\gamma$-ray photons with the same parent population spectrum and an energy $E_{\gamma} \approx 10 \% E_{p}$. However, also high energy electrons contribute to $\gamma$-ray emission through Bremsstrahlung and Inverse Compton processes. The distinction between leptonic and hadronic contribution is possible only if we detect typically hadronic features in the $\gamma$-ray spectrum.

The first one is at the lowest energies, where the rest mass of the $\pi^{0}, E_{\pi^{0}}^{0}=135 \mathrm{MeV}$, entails the presence of a "pion bump" at $E=E_{\pi^{0}}^{0} / 2(\sim 67.5 \mathrm{MeV})$. The second is at the highest energies: protons with $E \geq 10^{15} \mathrm{eV}$ imply photons with energies $E \geq 10^{14} \mathrm{eV}$ and a detection of $\gamma$-ray emission at this energy is definitely hadronic.

Detection of $\gamma$-ray emission spectrum below $200 \mathrm{MeV}$ from the SNR W44 by AGILE [28, 20] and Fermi-LAT [3] satellites seemed to be the long-awaited proof (see Sec.2). However, because of "ad hoc" acceleration model features in disagreement with theoretical previsions and of the high age ( $>10^{4} \mathrm{yrs}$ ) of this (and similar) remnant (Sec.2), diffusive shock re-acceleration of pre-existing CRs was taken into account $[1,31,21]$ (Sec.3). This mechanism could explain the $\gamma$-ray spectral behavior of middle-aged SNRs without any "ad hoc" feature (Sec.3.2). Moreover, DSRe could be present in the stellar wind Forward Shock (FS), as in the $\kappa$-Ori star in the Orion constellation [33, 22] (Sec.3.3) and could explain some features of secondary particle spectra [16] (Sec.3.4).

\section{The low-energy breakthrough: acceleration models and their problems}

After several years without any detection of $\gamma$-ray emission from SNRs at the lowest and at the highest critical energies, in 2011 there was a breakthrough. The AGILE satellite observed the middle-aged ( $t_{\text {age }} \geq 10000$ ) SNR W44, a very radio bright source, interacting with a Molecular Cloud (MC). Fermi-LAT observed this remnant already in 2010 [1] but its spectrum spanned down only to $E \sim 300 \mathrm{MeV}$. The AGILE spectrum, instead, extended down to $70 \mathrm{MeV}$ showing for the first time, even if not directly the "pion bump", a low energy decay in the Spectral Energy Distribution (SED) spectrum unquestionably due to hadronic emission [28, 20]. This behavior was the first direct evidence of CR energization in a SNR. In the following, after an improvement on its analysis software, Fermi-LAT satellite, not only confirmed the AGILE results for W44, but detected below $100 \mathrm{MeV}$ other two middle-aged SNRs, IC443 [3] and W51c [30]. 
The scientific community was so excited for these new results that it just assumed that these $\gamma$-ray spectra were due to freshly accelerated particles. Consequently, several acceleration models were developed in order to fit data. But are these models in agreement with theoretical previsions? The theory of DSA provides a power-law distribution for CR population with a momentum injection index $\alpha \leq 4$ in a strong shock (Mach Number $>>1$ and compression ratio $r_{s h} \leq 4$ ), depending on the non-linear effect of CRs on the shock $[12,9,14]$ :

$$
f_{i}(p)=k_{i}\left(\frac{p}{p_{\text {inj }}}\right)^{-\alpha}
$$

The normalization $k_{i}$, where the index $i$ indicates the particle specie, is proportional to CR acceleration efficiency, $\xi_{C R}$, to medium density, $\rho$, and to the square of the shock velocity, $v_{s h}$. This power-law distribution has a cut-off because the acceleration time has to be lower than he minimum between the age of the source and the loss time, $t_{a c c}=\frac{D(p)}{v_{s h}^{2}}<\min \left(t_{\text {loss }}, t_{\text {age }}\right) . D(p)$ is the diffusion coefficient and the losses are pp-emission and ionization for protons and heavy nuclei, and synchrotron, bremsstrahlung and inverse Compton for electrons [17, 21]. From the diffusion coefficient dependences [21], we obtain a general formula for the maximum momentum:

$$
p_{\max } \propto\left(B_{0}\right)\left(v_{s h}\right)^{\frac{2}{1-\delta}}\left(t_{\min }\right)^{\frac{1}{1-\delta}}\left(L_{c}\right)^{-\frac{\delta}{1-\delta}} .
$$

where $B_{0}$ is the upstream magnetic field, $\delta$ is the magnetic perturbation spectral index and $L_{c}$ is the magnetic perturbation correlation length [21, 22].

However, in order to explain the AGILE and Fermi-LAT data from the middle aged SNRs, no acceleration model can fit the spectra with a simple power-law with a cut-off $[28,20,3,30]$. All models add a low-energy cut-off or a break in the power-law distribution, using a high-energy index very steep, $\alpha \geq 3$. All features with a no simple explanation in the context of the DSA theory. There could be some physical explanations for these tricky behaviors. The low-energy cut-off could be explained by the failure of the low-energy CRs to penetrate the high density MC interacting with the remnants [26]. The steepening of the spectrum at high-energy, instead, could be due to the damping of Alfvén waves [32]. However, there is no explicit agreement with the theory.

There is, then, another important issue. The middle-aged SNRs like W44, IC443 and W51c, are in their advanced expansion phase (Sedov-Taylor phase) with a very low shock velocity $\left(v_{s}<\right.$ $100 \mathrm{~km} / \mathrm{s}$ ). Considering the relation between the normalization of CR acceleration spectrum, $k_{i}$, and $v_{s h}$, a slow shock implies a too high acceleration efficiency, $\xi_{C R}$, in order to explain the high $\gamma$-ray.

In the light of these difficulties, there were some attempts to explain the observed $\gamma$-ray spectra from these remnants taking into account the contribution from diffusive shock re-acceleration (DSRe) of pre-existing CRs.

\section{Reacceleration Contribution}

\subsection{Differences between re-acceleration and acceleration}

The first difference between DSRe and DSA is particle injection. For a pure acceleration, we need of a still unknown injection mechanism that enhances the energy of thermal particles enough 
for allow the first order Fermi acceleration at the shock [18, 21]. Re-acceleration, instead, takes high energy particles from pre-existing, almost isotropic, CR sea in our Galaxy. The spectrum of these particles is measured at the highest energies by different instruments as PAMELA and AMS02 [4, 5, 6, 7]. At the lowest energies, instead, we had "direct" measurements only after the Voyager spacecraft went beyond the heliosphere [43, 37, 38, 11]. Beyond the heliosphere the Sun influence is very low and CR spectrum is not affected by solar modulation.

The energization mechanism is the first order Fermi energization both for re-acceleration and acceleration. However, whereas for the acceleration we have the power-law spectrum in Eq.2.1, the spectrum for re-accelerated particles, $f_{0}(p)$, is obtained from the pre-existing CR spectra:

$$
f_{0}(p)=\alpha\left(\frac{p}{p_{m}}\right)^{-\alpha} \int_{p_{m}}^{p} \frac{d p^{\prime}}{p^{\prime}}\left(\frac{p^{\prime}}{p_{m}}\right)^{\alpha} f_{\infty}\left(p^{\prime}\right)
$$

where $p_{m}$ is the minimum momentum in Galactic CR spectrum and $f_{\text {infty }}(p)$ is the Galactic CR spectrum obtained from the fluxes of pre-existing CRs $[37,38,11]$. The effect of re-acceleration is to enhance particle momentum and to harden CR initial spectrum if this is steeper than $\alpha$ or to keep it equal to the parent population if it is harder than $\alpha$.

Another difference is the normalization. In the DSA, we have seen that it depends on shock velocity, medium density and acceleration efficiency. In the DSRe, instead, the normalization is correlated with pre-existing CR normalization and with the compression ratio of the shock (see Eq.3.1). Consequently, we have fewer free parameters and the maximum reachable flux is more constrained than in the acceleration case. For this reason, DSRe contribution is often sub-dominant respect the DSA one, that remains the main energization mechanism in the Galaxy.

In presence of a high density target, as in our middle aged SNRs W44, IC443 and W51c, the situation is a little more complex. If the target density is sufficiently high, an adiabatic compressed thin, totally ionized, shell can form in the downstream region ('crushed cloud' model) [13, 21, 23]. In this shell, energized CRs are further compressed through a compression factor $s$ correlated to the ratio between the target density and the downstream density [21]. The adiabatic compression can be limited only by the the maximum between thermal and magnetic pressure and it can enhance the normalization of re-accelerated spectrum enough to become the dominant contribution to the $\gamma$-ray emission.

\subsection{Supernova Remnants}

Since its importance in the context of CR topic, the SNR W44 was the first source for which re-acceleration hypothesis was tested. In the work of Uchiyama et al. [42], all the $\gamma$-ray detected middle aged SNRs (W44, W51c and IC443) were analyzed in a re-acceleration context. The authors used the parametrization of pre-existing Galactic CRs based on EGRET and BESS spectrometer measurements [40,39]. The same was done by Lee et al. [31] but considering the temporal evolution of the remnant. They take into account both primary and secondary particle emission with hadronic and leptonic energy losses. Their results show that re-acceleration and adiabatic compression can explain the detected W44 spectrum. However, in these models were applied a low-energy cut-off and a spectral steepening likely due to Alfvén damping [32], the same "ad hoc" features used in the several acceleration models. In Tang et al. [41], re-acceleration and adiabatic compression can explain the W44 $\gamma$-ray spectrum without any low-energy cut-off or spectral break. 
However, the authors do not consider energy losses and leptonic contribution, that instead could be very important.

Up to now, a more complete and updated model was the one described in Cardillo et al. [21]. For the first time, pre-existing CR parametrization from Voyager I data was used [43, 37, 38, 11] for protons, helium nuclei and electrons. Energy losses and secondary emission from all particles were considered. This model explains the radio and $\gamma$-ray spectra of the SNR W44 without any "ad hoc" feature but only with a high-energy cut-off due to the time scale relation (see Sec.2)[21, 22]. The final spectrum is shown in Fig.1 (left), where even a possible contribution from freshly accelerated CRs is considered and results sub-dominant.

This model can be applied every time there are the right conditions for particle energization at a shock of a source, with or without the presence of a "crushed-cloud". Re-acceleration, indeed, is always present: we only can compute if it is dominant or sub-dominant respect the pure acceleration.

\subsection{Stellar Wind Forward Shocks}

In the last years, both Fermi-LAT and AGILE satellites had analyzed the $\gamma$-ray diffuse emission in different regions of our Galaxy, in particular in the Orion region [2, 33]. The high energy diffuse emission is a combination of hadronic emission (pp and Bremsstrahlung) from $\mathrm{HI}$ and $\mathrm{H}_{2}$ distributions, leptonic emission (inverse Compton), isotropic extra-galactic emission and point-like source emission. These measurements were very useful in order to give an estimation of the CO to $\mathrm{H}_{2}$ conversion factor $X_{C O}$ but these entail another interesting result.

The study of the residual $\gamma$-ray emission obtained with the subtraction of the $\gamma$-ray diffuse one showed the presence of an excess near the OB star $\kappa$-Ori, both in the Fermi-LAT and AGILE data. There could be three different explanations for this excess: the "dark gas" emission [29], a possible non-linear relation between $\mathrm{H}_{2}$ and $\mathrm{CO}$ densities or $\mathrm{CR}$ acceleration from stellar wind shock. The "dark gas" contribution results too small to explain the measured flux of the excess [2,33]. Even if a non-linearity in the $X_{C O}$ estimation cannot be excluded, however, in the AGILE work was shown that there is a good correlation between the $\gamma$-ray excess and the $\mathrm{CO}$ distribution correspondent to a star formation region [36]. Consequently, the possibility of accelerated CR presence was studied $[22,23]$. Since the forward shock of $\kappa$-Ori star is very slow $\left(v_{s h} \sim 10 \mathrm{~km} / \mathrm{s}\right)$, however, an efficient acceleration was unlikely. A re-acceleration model, instead, both with or without the compressed shell formation this time, can explain very well the AGILE detected $\gamma$-ray spectrum (see Fig.1, right).

Actually, even a simple Blandford-like adiabatic compression could explain the AGILE spectrum [22]; nevertheless, the potential of pre-existing re-acceleration in sources other than SNRs is unquestionable.

\subsection{Beyond the $\gamma$-ray astronomy}

The role of pre-existing CRs DSRe was analyzed even separately from the $\gamma$-ray astronomy. The huge amount of data collected during the last decade, indeed, has unveiled a lot of tricky features in CR particle spectra $[15,8,27]$. One of this feature is the hardening of both primary and secondary spectra detected by PAMELA and AMS-02 above a rigidity of $300 \mathrm{GV}$ [16, 23]. 

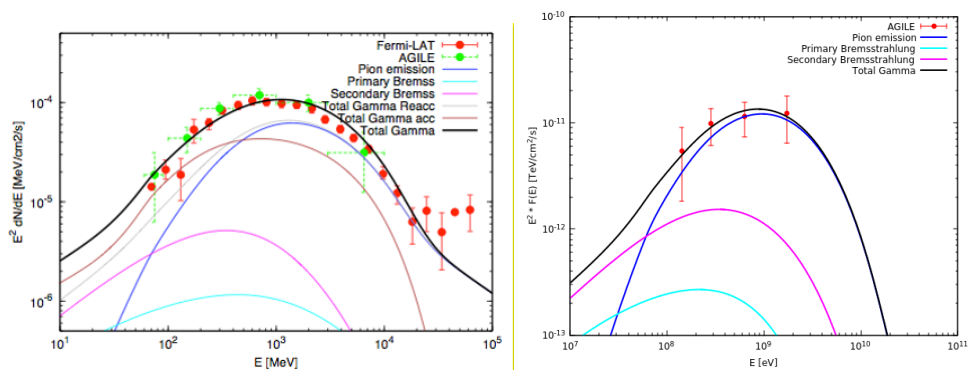

Figure 1: Left: $\gamma$-ray (AGILE and Fermi-LAT) data from W44 fitted with re-acceleration model described in [21]. Right: AGILE (red) $\gamma$-ray points plotted with the different contributions estimated re-acceleration model described in [22].

Secondary particles have a spectrum steeper of a factor $E^{-\delta}$ compared the primary ones. For this reason, the hardening-effect due to reacceleration (see Sec.3) strongly affect their spectra that become flatter and similar to primary particle spectra, entailing the $\mathrm{B} / \mathrm{C}$ ratio rising evident in the experimental data [16]. However, the hardening in primary particle spectra cannot be explained by re-acceleration and need another interpretation.

Last but not least, the introduction of pre-existing CRs in two-dimensional hybrid simulations has provided interesting results $[19,23]$. Previous simulations without DSRe showed the importance of the angle between the shock normal and the magnetic field in CR acceleration efficiency. Greater the angle (quasi-perpendicular shocks) lower the acceleration efficiency (inhibition of particle injection). Taking into account the DSRe in simulations, instead, it is shown that pre-existing CRs contribute in active way to self-generated turbulence, implying a non-linear magnetic filed amplification that changes shock inclination. This means that, even if the shock is quasi-perpendicular at the beginning, DSRe of pre-existing particles can modify its inclination and favor CR acceleration [19].

\section{Conclusions}

We have summarized the last results obtained in the context of CR energization through the consideration of diffuse shock re-acceleration of pre-existing particles. This mechanism is present in a source shock every time there are the right conditions for particle energization because involves particle from the Galactic CR sea. Its contribution is generically sub-dominant respect the pure acceleration. However, in slow shock as the ones in middle-aged SNRs or in the FS of the OB stars, the acceleration is inefficient and re-acceleration can become the main contribution to detected $\gamma$ ray spectra, especially in presence of a SNR-MC interaction.

\section{References}

[1] Abdo, A. A., Ackermann, M., Ajello, M. et al., Gamma-Ray Emission from the Shell of Supernova Remnant W44 Revealed by the Fermi LAT, Science 327 (2010) 1103

[2] Ackermann, M., Ajello, M., Allafort, A. et al., Gamma-Ray observations of the Orion Molecular Clouds with the Fermi-Large Area Telescope, ApJ 756 (2012) 4 
[3] Ackermann, M., Ajello, M., Allafort, A. et al. Detection of the Characteristic Pion-Decay Signature in Supernova Remnants, Science 339 (2013) 807-811

[4] Adriani, O.; Barbarino, G. C.; Bazilevskaya, G. A. et al. Measurements of Cosmic-Ray Proton and Helium Spectra, Science 332 (2011) 69

[5] Aguilar, M. et al., Electron and Positron Fluxes in Primary Cosmic Rays Measured with the Alpha Magnetic Spectrometer on the International Space Station, PRL 113 (2014) 121102

[6] Aguilar, M. et al. Precision Measurement of the Proton Flux in Primary Cosmic Rays from Rigidity 1 GV to 1.8 TV with the Alpha Magnetic Spectrometer on the International Space Station, PRL, 114 (2015) 171103

[7] Aguilar, M. et al. Precision Measurement of the Helium Flux in Primary Cosmic Rays of Rigidities 1.9 GV to 3 TV with the Alpha Magnetic Spectrometer on the International Space Station, PRL 115 (2015) 211101

[8] Amato, E., The origin of galactic cosmic rays, IJMPD 23 (2014) 7

[9] Bell, A.R., The acceleration of cosmic rays in shock fronts. i., Royal Astronomical Society 182 (1978a) 147

[10] Bell, A.R., The acceleration of cosmic rays in shock fronts. ii., Royal Astronomical Society 182 (1978b) 443

[11] Bisschoff, D. \& Potgieter, M. S New local interstellar spectra for protons, helium and carbon derived from PAMELA and Voyager 1 observations, AP\&SS 361 (2016) 48

[12] Blandford, R. D \& Ostriker, J. P., Particle acceleration by astrophysical shocks, ApJ 221 (1978) 29

[13] Blandford, R.D.; Cowie, L.L. Radio emission from supernova remnants in a cloudy interstellar medium, ApJ 260 (1982) 625-634

[14] Blasi, P. Nonlinear shock acceleration in the presence of seed particles, Astrop. Phys. 21 (2004) 45-57

[15] Blasi, P., The origin of galactic cosmic rays, A\&A 21 (2013) 70

[16] Blasi, P., On the spectrum of stable secondary nuclei in cosmic rays, MNRAS 471 (2017) 1662,

[17] Blumenthal, G.R. \& Gould, R.J., Bremsstrahlung, Synchrotron Radiation, and Compton Scattering of High-Energy Electrons Traversing Dilute Gases, Rev. Mod. Phys. 237 (1970) 42

[18] Caprioli, D. \& Spitkovsky, A., Simulations of Ion Acceleration at Non-relativistic Shocks. I. Acceleration Efficiency, ApJ 783 (2014) 91

[19] Caprioli, D., Zhang, H. \& Spitkovsky, A., Diffusive shock re-acceleration, J. Plasma Phys. 84 (2018) 7101

[20] Cardillo, M., Tavani, M., Giuliani, A. et al. , The supernova remnant W44: Confirmations and challenges for cosmic-ray acceleration, A\&A 565 (2014) 74

[21] Cardillo, M., Amato, E. \& Blasi, P., Supernova Remnant W44: a case of cosmic-ray reacceleration, A\&A 595 (2016) A58

[22] Cardillo, M., Marchili, N., Piano, G. et al., The Orion Region: Evidence of enhanced cosmic-ray density in a stellar wind forward shock interaction with a high density shell, A\&A 662 (2019) A57

[23] Cardillo, M., The Important Role of Cosmic-Ray Re-Acceleration, Galaxies 7, 2, 49662 (2019) A57 
[24] Fermi, E., On the origin of the Cosmic Radiation, Phys.Rev, 75, (1949) 1169-1174

[25] Fermi, E., Galactic Magnetic Fields and the Origin of Cosmic Radiation, ApJ 119 (1954) 1

[26] Gabici, S, Gamma-ray emission from supernova remnants and surrounding molecular clouds, AIPC (2017) 1792b0002G

[27] Gabici, S., Evoli, C., Gaggero, D. et al., The origin of Galactic cosmic rays: challenges to the standard paradigm, 2019arXiv190311584G (2019)

[28] Giuliani, A., Cardillo, M., Tavani, M., et al. 2011, Neutral Pion Emission from accelerated protons in the Supernova Remnant W44, ApJ 742 (2011) 30-34

[29] Grenier, I. A., Casandjian, J.-M., \& Terrier, R., Unveiling Extensive Clouds of Dark Gas in the Solar Neighborhood, Science 307 (2005) 1292

[30] Jogler, T. \& Funk, S. Revealing W51C as a cosmic ray source using Fermi-LAT data, ApJ 816 (2016) 100

[31] Lee, S.H., Patnaude, D.J., Raymond, J.C., Nagataki, S., Slane, P.O. \& Ellison D.C., Modeling bright $\gamma$-ray and radio emission at faust cloud shock, ApJ 206 (2015) 71

[32] Malkov, M.A., Diamond P.H. \& Sagdeev, R.Z., Mechanism for spectral break in cosmic ray proton spectrum of supernova remnant W44, NatCo 2 (2011) 194-198

[33] Marchili, N., Piano, G., Cardillo, M. et al., A new gamma-ray source unveiled by AGILE in the region of Orion, A\&A 615 (2018) 82

[34] Morlino, G., Bandiera, R., Blasi, P. \& Amato, E., Collisionless Shocks in a Partially Ionized Medium. II. Balmer Emission, ApJ 760 (2012) 137

[35] Morlino, G., Blasi, P., Bandiera, R. \& Amato, E., Cosmic ray acceleration and Balmer emission from $R C W 86$ (G315.4 - 2.3), A\&A 562 (2014) 141

[36] Pillitteri, I., Wolk, S.J. \& Megeath, S.T., A Star-forming ring around $\kappa$-Ori 250 pc from the sun, ApJL 820 (2016) L28

[37] Potgieter, M. S.; Vos, E. E.; Nndanganeni, R. R. et al., A Very Local Interstellar Spectrum for Galactic Electrons , arXiv1308.1666P (2013)

[38] Potgieter, M.S. Very Local Interstellar Spectra for Galactic Electrons, Protons and Helium, BrJPh 44 (2014) 581-588

[39] Shikaze, Y., Haino, S., Abe, K., et al., Measurements of $0.220 \mathrm{GeV/n}$ cosmic-ray proton and helium spectra from 1997 through 2002 with the BESS spectrometer, Astrop. Phys., 28 (2007) 154

[40] Strong, A.W., Moskalenko, I.V. \& Reimer, O., Diffuse Galactic Continuum Gamma Rays: A Model Compatible with EGRET Data and Cosmic-Ray Measurements, ApJ, 613 (2004) 692

[41] Tang, X. \& Chevalier, R.A., Time-dependent Diffusive Shock Acceleration in Slow Supernova Remnant Shocks, ApJ, 800 (2015) 103

[42] Uchiyama, Yasunobu; Blandford, Roger D.; Funk, Stefan et al., Gamma-ray Emission from Crushed Clouds in Supernova Remnants, ApJ 723 (2010) 122

[43] Webber, W.R. \& McDonald, F.B. Recent Voyager 1 data indicate that on 25 August 2012 at a distance of 121.7 AU from the Sun, sudden and unprecedented intensity changes were observed in anomalous and galactic cosmic rays, Geophys. Res. Lett. 40 (2013) 1665-1668 